\title{
Incidence of Atrioventricular Block After Valve Replacement in Carcinoid Heart Disease
}

\author{
Igor Sunjic ${ }^{a, f, g}$, Doosup Shin ${ }^{\text {b, f, Katlynd M. Sunjic }}{ }^{c}$, Jesal V. Popat ${ }^{\mathrm{a}}$, \\ Thanh Tran ${ }^{a}$, Sanders H. Chae ${ }^{a}$, Christiano C. Caldeira ${ }^{\text {, }}$ \\ Jonathan R. Strosberg ${ }^{\mathrm{e}}$, Dany Sayad ${ }^{\mathrm{a}}$
}

\begin{abstract}
Background: Carcinoid heart disease (CaHD) is a rare condition that has a high impact on the morbidity and mortality of its patients. Once heart failure symptoms develop in the patient with CaHD, cardiac valve surgery is often the only effective treatment. Although atrioventricular block (AVB) is a known postoperative complication of the valve surgery, the incidence of AVB in this population has not been well described.
\end{abstract}

Methods: Comprehensive records were collected retrospectively on consecutive patients with CaHD who underwent a valve surgery at a tertiary medical center from January 2001 to December 2015. We excluded patients with pre-existing permanent pacemaker (PPM).

Results: Nineteen consecutive patients were included in this study and 18 of them underwent at least dual valve (tricuspid and pulmonary valve) replacement surgery. Our 30-day post-surgical mortality was $0 \%$. During the 6-month observation period following the surgery, $31.5 \%(n=6)$ required PPM implantation due to complete AVB. There was no statistical difference in baseline characteristics and electrocardiographic and echocardiographic parameters between the patients who did or did not require PPM placement.

Conclusions: Our study revealed that almost one-third of CaHD patients who underwent a valve replacement surgery developed AVB requiring PPM implantation. Due to high incidence of PPM require-

Manuscript submitted November 3, 2019, accepted November 15, 2019

aDivision of Cardiovascular Sciences, University of South Florida Morsani College of Medicine, Tampa, FL 33606, USA

bDivision of Cardiovascular Medicine, University of Iowa Carver College of Medicine, Iowa City, IA 52242, USA

'Department of Pharmacotherapeutics and Clinical Research, University of South Florida College of Pharmacy, Tampa, FL 33606, USA

${ }^{\mathrm{d}}$ Division of Cardiothoracic Surgery and Cardiology, Tampa General Hospital, Tampa, FL 33606, USA

eDepartment of Gastrointestinal Oncology, H. Lee Moffitt Cancer Center and Research Institute, Tampa, FL 33612, USA

fThese authors contributed equally to this work.

${ }^{g}$ Corresponding Author: Igor Sunjic, Department of Cardiovascular Sciences, University of South Florida Morsani College of Medicine, 2 Tampa General Circle, STC 5th Floor, Tampa, FL 33606, USA. Email: tsunjic@gmail.com

doi: https://doi.org/10.14740/cr986 ment, we believe that prophylactic placement of an epicardial lead during the valve surgery can be helpful in these patients to reduce serious complication from placement of pacemaker lead on a later date through a prosthetic valve.

Keywords: Carcinoid heart disease; Atrioventricular block; Pacemaker; Valve disease; Valve replacement surgery

\section{Introduction}

Carcinoid heart disease (CaHD) is a fibrotic valvular process that occurs in patients with longstanding carcinoid syndrome and highly elevated levels of circulating serotonin [1]. Prior to development of effective systemic treatments for reduction of circulating serotonin levels, CaHD was reported to develop in approximately $50 \%$ of carcinoid syndrome patients [2]. In $\mathrm{CaHD}$, the right-sided heart valves are primarily affected because of their direct exposure to circulating blood serotonin, leading to right heart failure. Once patients with CaHD develop heart failure symptoms, the only effective treatment option is valve surgery, which has shown to decrease mortality and improve functional symptoms [3-8]. Majority of those patients required both tricuspid valve (TV) and pulmonary valve (PV) surgeries, preferably replacement [7, 9]. In 1995, Connolly and colleagues reported that 30-day mortality in CaHD patients undergoing cardiac surgery was $35 \%$ [3]. Subsequent studies have demonstrated a decline in overall operative mortality to $10 \%$ or lower after $2000[7,10]$.

A potential complication of the valve surgery for CaHD patients is atrioventricular block (AVB). However, prior studies have not focused on the morbidity associated with this event and the appropriate management of this complication. We therefore sought to examine the incidence of AVB and permanent pacemaker (PPM) implantation following valve replacement in $\mathrm{CaHD}$ patients.

\section{Materials and Methods}

This retrospective chart review was conducted on consecutive patients with CaHD who were evaluated by a cardiothoracic surgeon for a valve replacement surgery at a tertiary medical 
center between January 2001 and December 2015. This study was approved by Institutional Review Board of University of South Florida and Tampa General Hospital, and was conducted in accordance with the ethical standards of the institutions and with the Helsinki Declaration.

CaHD was diagnosed with echocardiography and presence of valvular thickening associated with regurgitation or stenosis. Valve surgery was recommended in stable CaHD patients if they had functional symptoms consistent with worsening heart failure or severe valvular dysfunction. Diagnoses of comorbid conditions were determined by the International Statistical Classification of Diseases and Related Health Problems (ICD)-9 or ICD-10 codes, with the exception of coronary artery disease, which had to be confirmed by left heart catheterization. Pre- and post-surgical electrocardiogram (EKG) and transthoracic echocardiogram data were collected and reviewed by an independent cardiologist.

Cardiopulmonary bypass was preformed through midline sternotomy. Inflow arterial cannula was placed in the ascending aorta and outflow cannulas placed in the superior and inferior vena cava. Operation was performed on a beating heart at normothermia. Bioprosthetic valve was used due to favorable outcomes without need for lifetime anticoagulation [7]. The biggest valve possible on the pulmonic position was used in order to maximize the unload of the weak right ventricle (RV). Our valve of choice was a $29-\mathrm{mm}$ freestyle bioprosthesis. In order to fit the valve, the RV outflow track was consistently enlarged with a patch.

Difference in categorical and continuous variables was analyzed using Fisher's exact test or Chi-square test and $t$-tests, respectively. Data were presented as mean \pm standard deviation (SD) or as proportion (\%). All statistical analyses were performed using SAS version 9.4 (SAS Institute Inc., Cary, NC, USA) and STATA version 12.1 (Stata Corp., College Station, TX, USA). Reported probability values were two-sided and a $\mathrm{P}<0.05$ was considered statistically significant.

\section{Results}

In total, 27 patients were identified with CaHD seeking surgical evaluation for valve replacement during the study period. Among them, seven patients did not undergo surgery due to extensive metastatic disease or comorbid conditions. One patient was excluded due to the presence of a PPM upon initial preoperative evaluation. After these exclusions, 19 patients were finally included in this study.

Table 1 demonstrates characteristics of study participants. The average age was $60.4 \pm 1.9$ years old and $57.9 \%$ were male gender. All 19 patients had symptomatic heart failure (New York Heart Association class III/IV) and were on octreotide therapy prior to the surgery. Two patients had had prior valve replacement and underwent redo valve surgery as a result of worsening disease. Sixteen patients underwent dual valve replacement (TV and PV), two patients underwent triple valve replacement (TV, PV, and aortic valve), and the other one underwent PV replacement.

During the 6-month observation period following surgery, $31.5 \%(\mathrm{n}=6)$ required PPM implantation due to com- plete AVB. There was no statistically significant difference in demographic and comorbidity profile, pre-procedural EKG parameters, and pre- and post-procedural echocardiographic parameters between PPM and non-PPM groups. Also, there was no significant difference in type of surgery between the two groups $(\mathrm{P}=0.088)$. All two patients who underwent triple valve replacement developed AVB and required PPM placement, but number was too small to detect statistical difference. Our 30-day surgical mortality was $0 \%$. Two patients died during the follow-up period, approximately 4 months after the surgery.

Table 2 demonstrates characteristics of patients who developed AVB and required PPM placement following the valve surgery. The mean time to PPM implantation after the surgery was $6.3 \pm 0.5$ days (median, 6.5 days). Device interrogation done in at least 4 months after the PPM implantation revealed that all patients were still dependent to the PPM except one patient whose interrogation result was not available.

\section{Discussion}

The majority of CaHD patients develop heart failure symptoms as a result of tricuspid and pulmonary insufficiency leading to right heart chamber dilation. Once they develop heart failure symptoms, the only effective treatment option is a valve surgery, and both TV and PV are usually replaced [7, 10]. In our retrospective study that included 19 patients with CaHD who underwent valve surgery, most patients $(\mathrm{n}=18)$ had at least dual valve replacement (TV and PV), and 30-day operative mortality was $0 \%$. Incidence of complete AVB requiring PPM placement after the surgery was $31.5 \%$ during the 6-month follow-up.

The incidence of PPM requirement after TV operation has been known to be higher than that after other valve interventions [11]. During replacement of the TV, independent of valve size, stitches are placed through the TV annulus, which is in close proximity to the AV node. Despite all efforts to avoid surgical injury to the $\mathrm{AV}$ node, injury to the node or even edema of the surrounding tissue could cause temporary or permanent AVB. In our study, the incidence of PPM requirement after valve replacement in patients with $\mathrm{CaHD}$ was even higher than that after overall TV operations performed in the same hospital during 2014 - 2015 (unpublished data; 24\%) or other experienced centers $(21-22 \%)$ [11, 12]. Although number of cases from our study was not enough to detect statistical significance of such difference, it should be noticed that almost one-third of CaHD patients required PPM placement after the valve replacement. Part of the reason is that those patients required multiple valve replacement, and multivalve surgery is a well-known risk factor for post-operative PPM requirement [13]. In addition, valve replacement is known to be associated with significantly higher risk of post-operative PPM requirement compared with valve repair [13], and all of our patients received valve replacement surgery.

AVB after valve surgery can be transient and recovery of conduction usually occurs before the seventh postoperative day $[13,14]$. However, since there are risks of delayed PPM implantation (e.g. prolonged immobilization and hospital 
Table 1. Characteristic of Patients With Carcinoid Heart Disease Who Underwent a Valve Replacement Surgery According to PostSurgical Permanent Pacemaker Placement

\begin{tabular}{|c|c|c|c|c|}
\hline & \multirow{2}{*}{ Overall $(n=19)$} & \multicolumn{3}{|c|}{ Post-surgical PPM placement } \\
\hline & & $\operatorname{PPM}(n=6)$ & No PPM $(n=13)$ & P value ${ }^{a}$ \\
\hline \multicolumn{5}{|l|}{ Demographic and comorbidity profile } \\
\hline Male & $11(57.9)$ & $1(16.7)$ & $10(76.9)$ & 0.041 \\
\hline Hypertension & $12(63.2)$ & $1(16.7)$ & $11(84.6)$ & 0.010 \\
\hline Coronary artery disease $^{c}$ & $2(10.5)$ & $1(16.7)$ & $1(7.7)$ & 1.000 \\
\hline Atrial fibrillation & $7(36.9)$ & $4(66.7)$ & $3(23.1)$ & 0.129 \\
\hline Obesity $^{\mathrm{d}}$ & $4(21.1)$ & $0(0.0)$ & $4(33.8)$ & 0.255 \\
\hline NYHA class III/IV & $19(100)$ & $6(100)$ & $13(100)$ & - \\
\hline \multicolumn{5}{|l|}{ Pre-surgical EKG parameters } \\
\hline First degree AVB & $3(15.8)$ & $0(0.0)$ & $3(23.1)$ & 0.522 \\
\hline$\geq$ Second degree AVB & $0(0)$ & $0(0)$ & $0(0)$ & - \\
\hline RBBB & $3(15.8)$ & $0(0.0)$ & $3(23.1)$ & 0.517 \\
\hline Left fascicular block & $2(10.5)$ & $1(16.7)$ & $1(7.7)$ & 1.000 \\
\hline PR interval (ms) & $167.6 \pm 13.0$ & $158.8 \pm 9.8$ & $171.6 \pm 18.6$ & 0.660 \\
\hline QRS interval (ms) & $102.3 \pm 4.0$ & $104.2 \pm 10.7$ & $101.5 \pm 3.7$ & 0.765 \\
\hline \multicolumn{5}{|l|}{ Pre-surgical TTE parameters } \\
\hline $\operatorname{LVEF}(\%)$ & $54.6 \pm 1.2$ & $56.8 \pm 1.9$ & $53.5 \pm 1.5$ & 0.236 \\
\hline \multicolumn{5}{|l|}{ Post-surgical TTE parameters ${ }^{\mathrm{a}}$} \\
\hline $\operatorname{LVEF}(\%)$ & $55.6 \pm 2.2$ & $55.8 \pm 4.0$ & $55.5 \pm 2.8$ & 0.945 \\
\hline Reduced RV function & $7(43.8)$ & $3(50.0)$ & $4(40.0)$ & 1.000 \\
\hline RVSP (mm Hg) & $30.3 \pm 2.5$ & $36.0 \pm 5.1$ & $28.0 \pm 2.7$ & 0.161 \\
\hline Type of surgery & & & & 0.088 \\
\hline PVR & $1(6.3)$ & $0(0.0)$ & $1(7.7)$ & \\
\hline $\mathrm{PVR}+\mathrm{TVR}$ & $16(84.2)$ & $4(66.7)$ & $12(92.3)$ & \\
\hline $\mathrm{PVR}+\mathrm{TVR}+\mathrm{AVR}$ & $2(10.5)$ & $2(33.3)$ & $0(0.0)$ & \\
\hline Post-surgical mortality & $2(10.5)$ & $1(16.7)$ & $1(7.7)$ & 1.000 \\
\hline Operative (30 days) & $0(0.0)$ & $0(0.0)$ & $0(0.0)$ & - \\
\hline During 6-months follow up & $2(10.5)$ & $1(16.7)$ & $1(7.7)$ & 1.000 \\
\hline
\end{tabular}

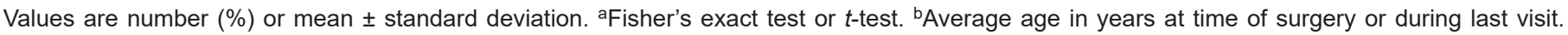
'Diagnosed via left heart catheterization. ${ }^{d}$ Body mass index $\geq 30 \mathrm{~kg} / \mathrm{m}^{2}$. AVB: atrioventricular block; AVR: aortic valve replacement; EKG: electrocardiogram; LVEF: left ventricular ejection fraction; ms: millisecond; NYHA: New York Heart Association; PPM: permanent pacemaker; PVR: pulmonary valve replacement; RBBB: right bundle branch block; RV: right ventricle; RVSP: right ventricular systolic pressure; TTE: transthoracic echocardiogram; TVR: tricuspid valve replacement. 
Table 2. Characteristics of Patients With Carcinoid Heart Disease Who Required Post-Surgical Pacemaker Placement

\begin{tabular}{llllllll}
\hline Patient & Surgery & $\begin{array}{l}\text { Year of } \\
\text { surgery }\end{array}$ & $\begin{array}{l}\text { Prior valve } \\
\text { surgery }\end{array}$ & $\begin{array}{l}\text { Reason } \\
\text { for PPM }\end{array}$ & $\begin{array}{l}\text { Time to } \\
\text { PPM (days) }\end{array}$ & PPM type & $\begin{array}{l}\text { PPM burden in } \\
\text { several months }\end{array}$ \\
\hline 1 & TVR, PVR & 2012 & Yes (TVR, PVR) & CHB & 6 & Dual-chamber & $100 \%$ V-paced \\
2 & TVR, PVR, AVR & 2014 & None & CHB & 8 & Dual-chamber & NA \\
3 & TVR, PVR & 2015 & None & CHB & 7 & Dual-chamber & $33 \%$ A-paced \\
4 & TVR, PVR & 2015 & None & CHB & 7 & Dual-chamber & $19 \%$ A-paced, 100\% V-paced \\
5 & TVR, PVR & 2004 & None & CHB & 5 & Dual-chamber & $100 \%$ V-paced \\
\hline 6 & TVR, PVR, AVR & 2012 & None & CHB & 5 & Dual-chamber & $87 \%$ A-paced, 11\% V-paced \\
\hline
\end{tabular}

AVR: aortic valve replacement; CHB: complete heart block; NA: not available; PPM: permanent pacemaker: PVR: pulmonary valve replacement; TVR: tricuspid valve replacement; A: atrial; V: ventricular.

stay), the best timing of permanent pacing is still controversial. Per literature review, it is generally recommended to defer the PPM placement no earlier than 5 - 7 days after the surgery [13-15]. In our study, the mean time to PPM implantation after the surgery was $6.3 \pm 0.5$ days (median, 6.5 days), which was not quite different from what was recommended. Furthermore, device interrogation done in several months after the PPM implantation revealed that those patients continued to be dependent on the PPM.

After TV replacement, placement of an endocardial ventricular pacemaker lead could be difficult. The lead, if placed at a later time through the prosthetic valve, will damage the prosthetic valve with time. It can also increase the risk of prosthetic endocarditis. To prevent such complications, preemptive placement of an epicardial right ventricular pacemaker lead was advised by our team. Therefore, all 19 patients in our study had a bipolar epicardial ventricular lead placed and tunneled underneath the left chest. These leads were well tolerated and relatively easy to connect if PPM needs to be utilized.

This study has several limitations. First, it was a singlecenter, retrospective study with a small sample size. Secondly, our study period extends over 14 years in which changes in the care of cardiac disease patients in general may have affected the results. Despite above limitations, this is the first study to investigate the incidence of AVB requiring PPM placement after valve replacement surgery in CaHD patients.

\section{Conclusions}

In conclusion, our study found that almost one-third of $\mathrm{CaHD}$ patients who underwent a valve replacement surgery required post-operative PPM placement due to AVB. Therefore, we believe that prophylactic placement of an epicardial lead during the valve surgery could be helpful in these patients to reduce serious complications that can result from repeat thoracotomy or placement of pacemaker lead on a later date through a prosthetic valve.

\section{Acknowledgments}

Parts of this study were presented at American Heart Associa- tion Scientific Sessions November 12 - 16, 2016, New Orleans, LA, USA.

\section{Financial Disclosure}

None to declare.

\section{Conflict of Interest}

All authors have nothing to disclose.

\section{Informed Consent}

This study was approved to waive informed consent process by the IRB committee.

\section{Author Contributions}

IS and D Sayad contributed to conceptualization; IS, D Shin, KMS, and TT contributed to data curation; IS, D Shin, KMS, and TT contributed to writing and original draft preparation; IS, D Shin, KMS, JVP, SHC, CCC, JRS, and D Sayad contributed to writing, critical review and editing.

\section{References}

1. Patel C, Mathur M, Escarcega RO, Bove AA. Carcinoid heart disease: current understanding and future directions. Am Heart J. 2014;167(6):789-795.

2. Pellikka PA, Tajik AJ, Khandheria BK, Seward JB, Callahan JA, Pitot HC, Kvols LK. Carcinoid heart disease. Clinical and echocardiographic spectrum in 74 patients. Circulation. 1993;87(4):1188-1196.

3. Connolly HM, Nishimura RA, Smith HC, Pellikka PA, Mullany CJ, Kvols LK. Outcome of cardiac surgery for carcinoid heart disease. J Am Coll Cardiol. 1995;25(2):410-416.

4. Moller JE, Pellikka PA, Bernheim AM, Schaff HV, Ru- 
bin J, Connolly HM. Prognosis of carcinoid heart disease: analysis of 200 cases over two decades. Circulation. 2005;112(21):3320-3327.

5. Bhattacharyya S, Raja SG, Toumpanakis C, Caplin ME, Dreyfus GD, Davar J. Outcomes, risks and complications of cardiac surgery for carcinoid heart disease. Eur J Cardiothorac Surg. 2011;40(1):168-172.

6. Manoly I, McAnelly SL, Sriskandarajah S, McLaughlin KE. Prognosis of patients with carcinoid heart disease after valvular surgery. Interact Cardiovasc Thorac Surg. 2014;19(2):302-305.

7. Connolly HM, Schaff HV, Abel MD, Rubin J, Askew JW, Li Z, Inda JJ, et al. Early and late outcomes of surgical treatment in carcinoid heart disease. J Am Coll Cardiol. 2015;66(20):2189-2196.

8. Edwards NC, Yuan M, Nolan O, Pawade TA, Oelofse T, Singh H, Mehrzad H, et al. Effect of valvular surgery in carcinoid heart disease: an observational cohort study. J Clin Endocrinol Metab. 2016;101(1):183-190.

9. Warner RRP, Castillo JG. Carcinoid Heart Disease: The Challenge of the Unknown Known. J Am Coll Cardiol. 2015;66(20):2197-2200.

10. Castillo JG, Filsoufi F, Rahmanian PB, Anyanwu A, Zacks JS, Warner RR, Adams DH. Early and late results of valvular surgery for carcinoid heart disease. J Am Coll Cardiol. 2008;51(15):1507-1509.

11. Jokinen JJ, Turpeinen AK, Pitkanen O, Hippelainen MJ, Hartikainen JE. Pacemaker therapy after tricuspid valve operations: implications on mortality, morbidity, and quality of life. Ann Thorac Surg. 2009;87(6):1806-1814.

12. Scully HE, Armstrong CS. Tricuspid valve replacement. Fifteen years of experience with mechanical prostheses and bioprostheses. J Thorac Cardiovasc Surg. 1995;109(6):1035-1041.

13. Koplan BA, Stevenson WG, Epstein LM, Aranki SF, Maisel WH. Development and validation of a simple risk score to predict the need for permanent pacing after cardiac valve surgery. J Am Coll Cardiol. 2003;41(5):795801 .

14. Meimoun P, Zeghdi R, D'Attelis N, Berrebi A, Braunberger E, Deloche A, Fabiani JN, et al. Frequency, predictors, and consequences of atrioventricular block after mitral valve repair. Am J Cardiol. 2002;89(9):1062-1066.

15. Kim MH, Deeb GM, Eagle KA, Bruckman D, Pelosi F, Oral H, Sticherling C, et al. Complete atrioventricular block after valvular heart surgery and the timing of pacemaker implantation. Am J Cardiol. 2001;87(5):649-651, A610. 